\title{
CUIDADOS EM SAÚDE: SOCIABILIDADES CUIDADORAS E SUBJETIVIDADES EMANCIPADORAS
}

\author{
CUIDADOS DE LA SALUD: SUBJETIVIDADES CUIDADORES Y DE \\ SOCIABILIDAD EMANCIPATORIA \\ HEALTH CARE: CAREGIVER SOCIABILITIES AND EMANCIPATORY \\ SUBJECTIVITIES
}

http://dx.doi.org/10.1590/1807-0310/2018v30177179

\author{
Octávio Augusto Contatore ${ }^{1}$, Ana Paula Serrata Malfitano ${ }^{2}$ e Nelson Filice de Barros ${ }^{1}$ \\ ${ }^{1}$ Universidade Estadual de Campinas, Campinas/SP, Brasil \\ ${ }^{2}$ Universidade Federal de São Carlos, São Carlos/SP, Brasil
}

\begin{abstract}
RESUMO
Embora predominem as aplicações técnico/tecnológicas na assistência à saúde como parâmetro e qualificação das ações de cuidado cresce a valorização de uma atenção que abranja mais amplamente a vida dos sujeitos, frente ao reconhecimento que o apoio social e a preocupação afetiva são constitutivos e fundamentais para a efetivação do cuidado. Objetivou-se apreender as reflexões sobre o cuidado à saúde enfocando os aspectos de sociabilidade e de subjetividade. Para tanto, realizou-se uma revisão sistemática de literatura, entre 2003 e 2013, em dez bases de dados. De 262 artigos identificados, foram selecionados 36 que enfocam a subjetividade nas ações de cuidado. Há um questionamento acerca do potencial de cuidado biomédico e suas limitações para atender às múltiplas demandas implícitas nos processos de saúde e doença. Conclui-se que a subjetividade e, em seu interior, a sociabilidade, são partes intrínsecas das ações de cuidado.
\end{abstract}

Palavras-chave: cuidados de saúde; apoio social; subjetividade.

\section{RESUMEN}

Aunque predominan las aplicaciones técnicas /tecnológicas en la asistencia sanitaria como un parámetro y calificación de las acciones de cuidado se reconoce cada vez más la atención que abarca más ampliamente la vida de los sujetos, con el reconocimiento de que el apoyo social y emocional para alguien son constitutivas y fundamental para la eficacia de el cuidado. Se objetivó aprehender las reflexiones sobre el cuidado a la salud enfocando los aspectos de sociabilidad y de subjetividad. Para tanto, se realizó una revisión sistemática de la literatura entre 2003 y 2013 en diez bases de datos. 262 artículos fueron identificados, 36 seleccionadas, los cuales se centran en la subjetividad en las acciones del cuidado. Hay una pregunta sobre el potencial de la atención biomédica y sus limitaciones para satisfacer las múltiples exigencias implícitas en la salud y la enfermedad. Se concluye que las subjetividad y, en su interior, la sociabilidad son partes intrínsecas de las acciones de cuidado.

Palavras-clave: cuidados en salud; apoyo social; subjetividad.

\begin{abstract}
Although technical/technological applications in health care predominate as a parameter and qualification of care actions, a wider approach regarding the life of the subjects is becoming more common, with the recognition that social support and emotional concern are constitutive and fundamental for an effective care. The research objective was to understand the reflections about health care focusing on sociability and subjectivity in care actions. Therefore, it was performed a systematic review of the literature between 2003 and 2013, on ten databases. 262 articles were identified and 36 of them selected for analysis. There is a questioning regarding the biomedical care potential and its limitations to answer the multiple demands implicit in health and disease. It is concluded that subjectivity and, in its interior, sociability are intrinsic parts of care actions.
\end{abstract}

Keywords: health care; social support; subjectivity. 


\section{Introdução}

Embora haja na atualidade uma multiplicidade de abordagens sobre o cuidado no campo da saúde, o referencial que as norteia é, predominantemente, o do modelo biomédico, que preconiza, em primeiro lugar, o tratamento à doença. Isso aconteceu porque o cuidado, no seu sentido mais estrito, de apoio social, desvelo e preocupação afetiva foi considerado de menor valor, enquanto que a sua expressão caracterizada por uma aplicação técnica racional e mediada pela tecnologia ganhou maior status social. No entanto, tem crescido a crítica à hegemonia do pensamento biomédico e tem sido fortalecida a discussão da importância de se oferecer outras formas de cuidado que contemplem o apoio social, as escolhas, os sentimentos, as emoções e os sentidos próprios que emergem de cada sujeito no seu adoecer. Ou seja, que contemplem a sociabilidade e a subjetividade para a efetivação das ações de cuidado.

Estudos empreendidos no campo das ciências sociais buscaram apreender como diferentes grupos sociais compreendem o que é saúde, doença e morte e quais são as formas que intervêm em tais processos. No século XX, autores como Foucault (1977), Canguilhem (2002), Illich (1975) e Boltanski (2004) voltaram sua atenção para a sociedade ocidental e sua medicina. Os seus estudos propiciaram uma visão crítica sobre como a ciência e a cultura moderna contribuíram para o desenvolvimento da clínica médica, da formalização da doença como uma entidade própria e da colonização do saber médico estendendo o seu domínio para tudo que diz respeito às ações de assistência à saúde.

Mais recentemente, estudos sociológicos ampliaram sua atenção para os profissionais sobre como exercem as ações de cuidado na assistência à saúde e como os receptores dessas ações as compreendem e se sentem contemplados por elas. Dentre as proposições elaboradas, houve a defesa da necessidade de uma maior integração entre as ciências sociais e a biomedicina, na formulação de um cuidado que considere, na análise da causalidade, ambos os aspectos implícitos no adoecimento: o social e o biológico (Pescosolido, 2006).

A diversidade de abordagens teóricas e metodológicas da sociologia da saúde produziu um corpo de conhecimento empírico sobre como fatores sociais, psicológicos, econômicos e culturais predispõem ao aparecimento da saúde e da doença (illness) e como as "doenças" (diseases) são muitas vezes o reflexo das atuais teorias diagnósticas e de seus procedimentos, bem como das interpretações culturais. Os estudos sociológicos demonstraram, também, como a qualidade e a quantidade de tratamentos não são claramente marcadas por certo perfil de doença e não são passíveis de um único conjunto de tratamentos. Além de que eventuais resultados de saúde, doença e cura são moldados por fatores extramédicos ou extratratamentos (Pescosolido, 2006).

$\mathrm{Na}$ interface entre as áreas da sociologia e da saúde, houve o desenvolvimento de novos referenciais para abordar o cuidado. Ampliaram-se os limites conceituais sobre o seu significado, sobre quem o recebe e como ele é aplicado. Mas, principalmente, ensejou a crítica ao olhar parcial do modelo biomédico de assistência à saúde e ao cuidado tecnicamente mais sofisticado, aparentemente o mais resolutivo, contrariando a lógica de que o melhor cuidado é o mais especializado e tecnológico (Giddens, 2005).

Dentro dessa discussão, este texto visou a apreender as reflexões sobre o cuidado à saúde pelo ponto de vista da literatura indexada, enfocando os aspectos de sociabilidade e de subjetividade nas ações de cuidado.

\section{Método}

Apresenta-se parte dos resultados encontrados na Revisão Sistemática da Literatura (RSL), intitulada: Os Sentidos do Cuidado em Saúde. Para efetuar a RSL, foram utilizados os descritores "Assistência à saúde" or "Prestação de Assistência à Saúde" or "Prestação de Cuidados de Saúde" or "Prestação de Assistência Odontológica" or "Prestação de Cuidados Odontológicos" or "Cuidados de Saúde" or "Cuidados de Assistência à Saúde" or "Rede de Cuidados Continuados de Saúde" and "Sociologia Médica" na Biblioteca Virtual em Saúde e seus correspondentes em inglês "Delivery of Health Care" or "Health Care Delivery" or "Health Care Systems" or "Delivery of Dental Care" or "Health Care" and "Sociology, Medical" nas bases de dados que contemplam artigos da área da saúde: PubMed, Scopus, Embase, Web of Science, Francis (OVID), ProQuest Central e Academic Search Premier (EBSCO HOST). E com os descritores Care or Health or Healthcare and (abstract) Sociology nas bases de dados que contemplam artigos das ciências sociais: Jstor e Sage.

Os critérios de inclusão foram: ter sido publicado no período entre 2003 e 2013; ser um artigo científico indexado; ser publicado em português, espanhol ou inglês; ter um dos descritores no título ou no resumo. Já os critérios de exclusão foram: artigos que não fossem de acesso aberto ou não disponibilizados no Portal de Periódicos da Coordenação de Aperfeiçoamento de 
Pessoal de Nível Superior (CAPES), artigos de revisão de literatura, carta, editorial, dissertação ou tese.

Foram realizadas quatro fases da busca nas bases de dados, a saber: (a) busca bruta em cada base filtrando apenas com a utilização de aspas pelos descritores, contabilizando 852 textos; (b) removeram-se os textos duplicados e aplicaram-se os critérios acima elencados, restando 262 textos; (c) leitura na íntegra dos artigos, eliminando 60 que não tinham nenhuma relação com a temática do cuidado; e (d) em nova leitura mais aprofundada, restringiu-se o universo àqueles que abordavam a temática cuidado de forma central em sua discussão, resultando em 63 textos. Esses foram classificados em cinco categorias temáticas, sendo que duas delas, contando com 36 artigos, nomeadas de Contemplar a subjetividade, sentimentos e emoções nas ações de cuidado e Cuidado centrado no paciente, foram utilizadas para este trabalho. Destaca-se que o uso da palavra "paciente" foi decorrente de sua predominância nos textos em estudo. $\mathrm{O}$ esquema do processo de seleção dos artigos está descrito na Figura 1.

\section{Figura 1: Processo de seleção dos artigos}

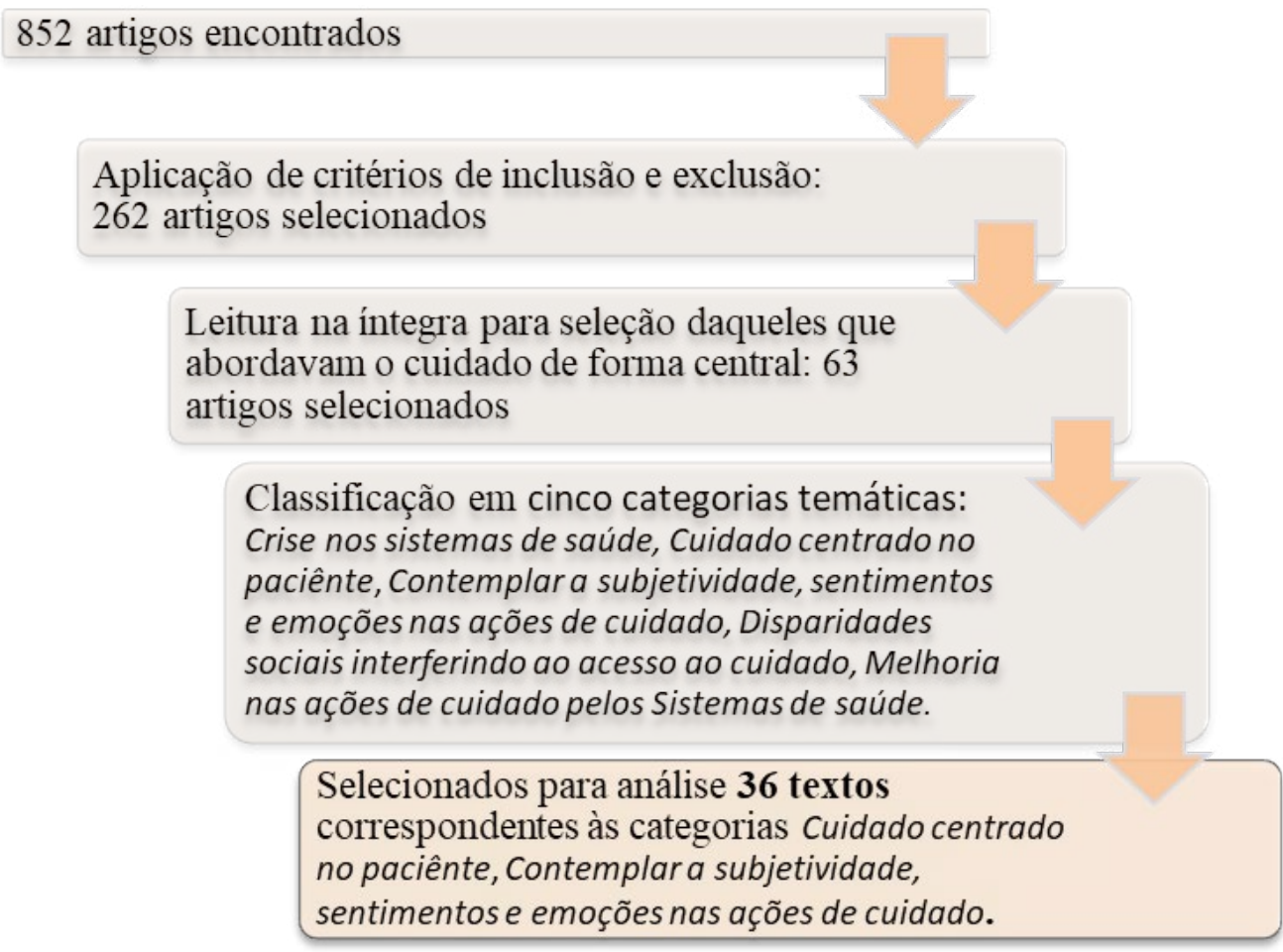

\section{Resultados e discussão}

Ao analisar os textos selecionados, emergiram quatro temáticas contemplando as questões de sociabilidade e subjetividade implícitas nas ações de cuidados. Houve textos que estavam presentes em mais de uma temática. As temáticas e suas respectivas referências foram: (a) as limitações do cuidado biomédico: Pescosolido, 2006; Brown e Flores, 2011; Burkhart e Hogan, 2008; Bahar et al., 2005; Edwards, Staniszweska e Crichton, 2004; Smith, 2009; Zimmermann e Rodin, 2004; (b) a sociabilidade nas ações de cuidado: Pescosolido, 2006; Carpentier, 2013; Carpentier e Grenier, 2012; Farrant e Watson, 2004; Denier, 2008; Dovidio et al., 2008; Ferlander, 2007; Fine, 2005 ; Greenhalgh, Robb e Scambler, 2006; Noone e Stephens, 2008; Prior, 2003; Salvalaggio et al., 2013; Sorensen e Iedema, 2008; Spates, 2012; Andersen et al., 2011; (c) o entrelaçamento entre cuidado e subjetividade: Pescosolido, 2006; Brown e Flores, 2011; Edwards, Staniszweska e Crichton, 2004; Zimmermann e Rodin, 2004; Carpentier, 2013; Basu, 2006; Hudson et al., 2006 ; Levy, 2005; Lopez, 2006; Schouffoer et al., 2011; Solheim e Garratt, 2013; Turner et al., 2003; Weeber, 2005; Wajid, White e Karim, 2013; (d) práticas integrativas e complementares: Daykin, McClean e Bunt, 2007; Freidin e Timmermans, 2008; Gyasi et al., 2011; Isola, 2013; Saizar e Korman, 2012; Shuval et al., 2012. 


\section{As limitações do cuidado biomédico}

Compõem este tema os trabalhos que fizeram crítica explícita ao modelo de produção de conhecimento da biomedicina, seu reflexo e limitações na aplicação de cuidado no campo da saúde.

Compreendeu-se que, apesar de gradativamente aumentar o desprestígio dos cuidados oferecidos pelo modelo clínico convencional, especialmente por abstrair o sujeito e polarizar a atenção no tratamento à doença, a biomedicina contemporânea continuamente recria a promessa de ofertar a mais eficaz assistência à saúde. Na atualidade, a Medicina Baseada em Evidências (MBE) e a validação de procedimentos pelo uso de Ensaios Clínicos Controlados (ECC) e estudos de coortes renderam legitimidade científica e pública para os profissionais da saúde continuarem a aperfeiçoar uma assistência protocolada na singularidade da doença, e não do doente (Tesser, 2007). No entanto, vários artigos selecionados fazem a crítica a essa padronização do cuidado (Bahar et al., 2005; Brown \& Flores, 2011; Burkhart \& Hogan, 2008; Edwards, Staniszweska, \& Crichton, 2004; Pescosolido, 2006; Smith, 2009).

Estudos sociológicos no campo da saúde problematizaram que existem diferenças entre a eficácia dos tratamentos que funcionam estritamente dentro de ensaios clínicos controlados e aqueles que funcionam no mundo real e, principalmente, não concordaram com a expectativa de que os tratamentos têm efeito idêntico e que as pessoas reagem da mesma forma. A complexidade da vida social e comportamental do indivíduo permite e requer o foco em diferentes níveis de análise e o uso de variadas abordagens de investigação. Como os métodos disponíveis para as ciências físicas e naturais de controle, manipulação e randomização não são eticamente possíveis para a maioria das pesquisas em ciências sociais e humanas, isso acarreta maiores desafios de investigação. Contudo, a diversidade de teorias, métodos e ferramentas analíticas utilizadas na área das ciências sociais favoreceram o desafio de "desembalar" o ambiente para a investigação biomédica (Pescosolido, 2006).

Estudos qualitativos, por exemplo, ajudaram a relativizar a capacidade da MBE em determinar a eficácia de procedimentos. Edwards, Staniszweska e Crichton (2004) entrevistaram ex-participantes de ECC que, além de avaliarem a eficácia de procedimentos para o tratamento de doenças, também aferiam a satisfação do paciente durante e após o seu tratamento. Nesses estudos, os autores constataram que houve influência de pressões psicológicas e sociais nos participantes, induzindo-os a responderem como satisfatórios seus tratamentos mesmo quando não o foram e a reinterpretarem positivamente experiências negativas. O que levou ao questionamento da real objetividade que os ECC pretendem demonstrar. Para Smith (2009), a alienação, tanto da medicina, da psiquiatria e da adoção da MBE, causaram muitas barreiras para a pesquisa de problemas complexos de saúde, por privilegiar especialmente o método quantitativo. Com isso, considerou-se que os ECC expressam um reducionismo biomédico, que tende para a racionalidade e objetividade e vê com maus olhos a subjetividade.

Quando se trata da atenção aos doentes terminais, por exemplo, a morte torna-se mais um evento técnico do que uma questão social-moral, ou seja, uma falha na tecnologia em resgatar o corpo. Ao não perceber a diferença entre prolongar a vida, para o que pode ser chamado apenas de prolongar a morte, a saúde passou de uma área humanitária e respeitada para uma nova e despersonalizada ciência (Zimmermann \& Rodin, 2004). Com isso, a clínica referenciada pela racionalidade científica objetivou as ações de cuidado e instituiu um modelo técnico/tecnológico de atenção que distanciou o profissional do paciente (Camargo, 2005; Tesser, 2007). A inversão de valores trazidos pelos métodos objetivamente orientados tem preconizado que as manipulações técnicas são mais claramente definidas do que são os seus efeitos nas pessoas e sua relação com valores humanos (Zimmermann \& Rodin, 2004).

Embora prevaleça a hegemonia de práticas de cuidado, que pesquisam e intervêm nas doenças reduzindo-as a um fato concreto, fixo e imutável, manifestados por sintomas no organismo (Tesser, 2007), simultaneamente cresceu a procura entre profissionais da saúde (Barros, 2000) e usuários (Giddens, 2005) por um modelo de atenção que ofereça um cuidado que não reduza o sujeito atendido à sua patologia e que abranja todas as questões envolvidas nos processos de saúde-doença-cuidado. Encontrouse, por exemplo, tal questão no artigo que aborda os profissionais da enfermagem, quando ressaltam a atenção e o respeito às influências culturais, familiares, de cunho espiritual e da comunidade na compreensão dos indivíduos (Bahar et al., 2005; Burkhart \& Hogan, 2008).

A clínica como prática de cuidado à saúde desenvolveu-se predominantemente em consonância com uma metodologia positivista da ciência moderna. A objetivação do saber científico contribuiu para uma reconfiguração do saber em saúde e, nesse processo, o doente e a sua subjetividade foram aos 
poucos destituídos de qualquer relevância para o conhecimento do fato patológico. A postulação de uma ciência positiva, objetal e factual levou o médico, influenciando posteriormente outros profissionais da saúde, a abstrair o paciente e colocá-lo entre parênteses, tornando-o apenas um fato exterior àquilo que o fazia sofrer. A clínica focou seu estudo na doença e descreveu-a minuciosamente, dando-lhe uma identidade própria; já o doente e as minúcias de sua história resumiram-se a itens úteis para a anamnese e entendimento do desenvolvimento da patologia (Canguilhem, 2002; Foucault, 1977; Illich, 1975).

Esse quadro, embora não único, permanece na atualidade, já que na clínica contemporânea a anamnese, os exames físicos e complementares buscam diagnosticar doenças, e o doente foi relegado a uma posição passiva de seu próprio processo. Uma prática focada na doença descarta qualquer responsabilidade pela história do sujeito concreto (Campos \& Bedrikow, 2014).

A presente hegemonia do conhecimento científico e a sua forma de perceber e conduzir o mundo pelo uso da razão produz pouca comunicação e diálogo com tudo que não se enquadre em seus parâmetros. Um dos impactos do modelo pragmático e utilitário das tecnociências, advindo da sua dominação cultural, é apresentar seu conhecimento como uma verdade única. No entanto, não oferece respostas para perguntas pertinentes à vida social, especialmente quando se trata de questões relacionadas à saúde humana (Luz, 2005).

\section{A sociabilidade nas ações de cuidado}

Sociabilidade é entendida aqui como espaços comunicacionais de interação entre grupos, rede e indivíduos em que se definem e redefinem simbolicamente certas diferenças socioculturais, que expressam possibilidades de construção temporária do próprio social (Frúgoli, 2007). A exemplo de Castel (1998), considera-se o pertencimento e a proteção derivados das redes familiares, de vizinhança e comunitárias como sociabilidade primária e os auxílios profissionais como sociabilidade secundária.

Os artigos selecionados neste item problematizaram a influência exercida pela interação entre sujeitos nas ações de cuidado no resultado dos tratamentos de saúde, sejam horizontais entre os membros da família e da comunidade, ou verticais entre o profissional de saúde e o paciente (Carpentier, 2013; Carpentier \& Grenier, 2012; Pescosolido, 2006). A qualidade dessas relações interferiu no resultado e na produção de autonomia nos cuidados à saúde, especialmente quando houve a valorização de uma maior participação dos sujeitos nas decisões que envolveram o seu cuidado (Carpentier, 2013; Carpentier \& Grenier, 2012; Fine, 2005; Prior, 2003).

A sociabilidade nas relações de cuidado foi abordada pelos estudos que compreenderam a força dos contextos sociais para o resultado positivo nos tratamentos de saúde. O apoio das redes sociais, das quais a pessoa faz parte, influenciaria mais o resultado do tratamento do que o acesso a um alto grau de recursos técnico/tecnológicos. Percebeu-se que a qualidade do cuidado não estava diretamente relacionada à expertise técnica de um profissional de saúde, mas, sim, ao suporte que o apoio social oferecia à pessoa que adoece (Carpentier, 2013; Carpentier \& Grenier, 2012; Pescosolido, 2006).

Do ponto de vista das relações verticalizadas, entre profissionais e usuários, postulou-se que o desgaste acumulado do modelo biomédico de cuidado à saúde acarretou, no final do século XX, uma crise de legitimação, desencadeando novas atitudes dos profissionais no relacionamento com o sujeito atendido, os quais passaram a valorizar um novo tipo de diálogo com o paciente (Greenhalgh, Robb, \& Scambler, 2006; Prior, 2003).

Essa abordagem trouxe a reformulação do paradigma da biomedicina convencional, afetando tanto os cuidados formais quanto os informais. Em lugar do padrão hierárquico do cuidado, como teorizado por Talcott Parsons (1951), emergiu uma concepção mais engajada e ativa de relacionamento, com base no reconhecimento dos direitos de ambas as partes como sujeitos. Cuidado, nesse sentido, precisou ser visto não apenas como uma atividade unidirecional realizada pelo cuidador, mas sim como o resultado da relação entre as diferentes partes, em que o respeito mútuo e o fomento das capacidades e da autonomia do destinatário vinham acima de tudo (Fine, 2005).

Para tanto, foi necessário avaliar democraticamente todas as formas de conhecimento, como também o status privilegiado do conhecimento científico em relação a outras formas de entendimento de saúde. Ao considerar que todas as formas de conhecimento são iguais em valor, postulou-se que o conhecimento não especializado tem a oferecer diferentes entendimentos dos processos de saúde, de doença e de cuidado, levando à democratização e maior participação do paciente nos processos decisórios (Prior, 2003).

Ao destacarem-se as estratégias de ação dos atores e seus processos de tomada de decisão, os comportamentos relacionados à não adesão 
aos tratamentos, à evasão dos serviços de saúde ou relutância em pedir ajuda foram mais bem compreendidos. Valorizaram-se as histórias pessoais daqueles que assumiram maior controle sobre suas próprias biografias e seus processos reflexivos, permitindo-os intervir e moldar o mundo como arquitetos de suas vidas. De forma que os arranjos sociais complexos e, às vezes, inesperados somam-se a cada história, imbuindo cada sujeito da construção de seus próprios significados (Carpentier, 2013).

A parceria entre cuidadores formais e informais, principalmente para melhor atender aos idosos, às pessoas com deficiência física ou mental e às crianças, deixou ver que os programas de assistência formal precisam contribuir para a manutenção da rede de cuidados informais, sobretudo sendo capazes de: criar espaços de diálogo e participação dos utilizadores do serviço, gerir desacordos, apoiar a existência de diversas opções e incentivar pequenas ações que aproximariam os membros das redes de cuidado (Carpentier, 2013).

O relacionamento com o paciente é potencializado quando os profissionais de saúde adotam estratégias de autonomia e de apoio, como o fornecimento de informações de uma forma não controladora e com empatia. A qualificação dos cuidados à saúde é influenciada pela presença ou não de empatia, escuta, vergonha, discriminação e flexibilidade (Salvalaggio et al., 2013). A não discriminação é fundamental para evitar danos no relacionamento entre os atores envolvidos; no entanto, é frequente a relação hierarquizada e estereotipada entre o profissional e o usuário, produzindo interações verticais que podem camuflar a discriminação e o preconceito implícitos, como aquelas que existem em função do gênero, do status social, do acesso aos bens culturais, da marginalização ou por influência de crenças (Denier, 2008; Dovidio et al., 2008; Ferlander, 2007; Noone \& Stephens, 2008; Spates, 2012).

Robb e Scambler (2006) apontam, dentre as dificuldades de comunicação entre profissional e paciente: a rapidez do atendimento; o foco nas questões biomédicas e consequente falta de escuta de relatos da vida pessoal; a falta de uma real concordância entre as partes, com predominância das opiniões em detrimento das questões relacionadas à vida do paciente; o predomínio da voz do conhecimento técnico à voz do mundo do paciente. A interação fica abaixo dos ideais teóricos de reciprocidade, confiança, partilha de poder, troca sincera de informação, reconhecimento do outro, com comprometimento da qualidade de tratamento e de cuidados.

\section{O entrelaçamento entre cuidado e subjetividade}

A subjetividade está relacionada à unidade psíquica, emocional e cognitiva do ser humano, portanto, está intrinsecamente ligada aos contextos sociais e culturais em que acontece a ação humana. Como um tema tanto da psicologia quanto das ciências sociais, é mais do que um sistema determinista intrapsíquico, evidenciando o contexto vivido no cotidiano (González Rey, 2007; Santos \& Martínez, 2016). Como afirma González Rey (2007, p. 172): "O sentido subjetivo não representa uma expressão linear de nenhum evento da vida social, pelo contrário, ele é o resultado de uma rede de eventos e de suas conseqüências colaterais ... produções de sentido subjetivo são inseparáveis da organização subjetiva atual dos sistemas humanos".

Destacam-se aqui os textos que buscaram compreender e realçar os aspectos emocionais, implícitos no comportamento dos sujeitos e dos profissionais. A importância dada aos aspectos subjetivos no cuidado favoreceu $\mathrm{o}$ aumento das investigações sobre o impacto psicológico na perspectiva de cada indivíduo ao ser submetido a um tratamento (Pescosolido, 2006), já que são vistos, medicados e tratados como problemas de saúde, sintomas físicos e psíquicos relacionados a processos emocionais (Basu, 2006). No entanto, novas propostas e considerações foram criadas com o intuito de favorecer o cuidado, sobretudo direcionadas a ajudar os técnicos e as instituições de assistência à saúde a expressar sentimentos e emoções em prol de uma atenção mais humanizada (Basu, 2006; Lopez, 2006).

Com o intuito de ajudar os profissionais da área da saúde a lidarem melhor com suas emoções e serem mais receptivos às emoções dos pacientes, Lopez (2006) relata sobre um novo constructo sociológico: o conceito de cuidado emocionalmente organizado. Visou a ampliar a atenção prestada por funcionários/ cuidadores para: atender às necessidades emocionais e sociais dos pacientes, manter a flexibilidade nas atividades internas, adaptando-as a necessidades emergentes, favorecer relações emocionais mais autênticas entre pacientes e cuidadores e observar a humanidade no cuidado, respeitando a pessoa além do corpo a ser tratado. Essas mudanças, quando experimentadas, criariam um ambiente mais acolhedor e menos estressante para profissionais e usuários.

Por outra via, a partir do campo da sociologia, Brown e Flores (2011) discutiram a importância de sentimentos como a compaixão e a esperança para o desenvolvimento de uma sociedade comprometida com o cultivo da justiça social e da dignidade humana. $\mathrm{Na}$ 
perspectiva dos autores, a diretriz do sistema de saúde, quando orientada para o mercado, pode negligenciar outras normas não técnicas para oferecer cuidados, como o altruísmo, a solidariedade e a compaixão.

Problematizou-se que aspectos subjetivos, presentes na assistência à saúde, nas mais variadas situações, contribuem para camuflar o real resultado obtido em um tratamento. Normalmente, apenas as principais consequênciasnão intencionais do tratamento são relatadas, tais como: mortalidade, morbidade ou medidas objetivas de gravidade da doença, enquanto outros resultados não são registrados, ou seja, aqueles que podem ter um grande impacto na qualidade de vida do paciente (Levy, 2005). Por outro lado, pressões psicossociais podem trabalhar em conjunto para quase padronizar a opinião dos entrevistados em pesquisas de satisfação após um tratamento. A primeira concerne à dominância do sistema de saúde, expressa na relação desigual entre profissional e paciente. A pessoa pode sentir que tudo o que tem a dizer ou pedir será julgado ou considerado de menor valor, o que o levaria a inibir suas perguntas e comentários. A segunda pressão vem de um tipo de etiqueta social, que leva a pessoa a não falar o que está pensando. A terceira pressão está relacionada a respostas psicológicas dos próprios pacientes em relação ao seu futuro, a preferirem olhar positivamente para ele (Edwards, Staniszweska, \& Crichton, 2004).

No estudo de Wajid, White e Karim (2013), demonstrou-se que as pessoas procuraram serviços mais distantes e caros, independente de sua situação econômica, quando perceberam que esses ofereciam melhores condições de cuidado. Verificaram tal fator entre os requisitos considerados importantes para uma melhor atenção à saúde reprodutiva das mulheres. Na atenção a idosos, segundo Carpentier (2013), na ausência de soluções claras e eficazes para seus problemas, a intervenção poderia ser enraizada na compreensão das trajetórias de vida e das redes familiares disponíveis dos pacientes. Para residentes de lares de idosos, a maior parte do sofrimento suportado por eles vem do tédio, da solidão e do desamparo, e não da dor física. Então, uma mudança de perspectiva de atuação nesses ambientes envolveria uma autocrítica organizacional com vista a identificar e implementar formas de abordar as necessidades emocionais e sociais dos residentes (Lopez, 2006). Ouvir as razões de pessoas que expressam o desejo de morrer poderia permitir ao profissional de saúde mobilizar estratégias terapêuticas adequadas e abordagens clínicas que visam a responder a essas preocupações, pois a abordagem necessária é aquela que procura compreender, ao invés de apenas atuar sobre o pedido. Um compromisso de comunicação sensível que poderia ajudar a descobrir o significado, para o paciente, do seu desejo (Hudson et al., 2006).

Isso também ocorre na aplicação dos cuidados paliativos e no confronto com a impossibilidade de cura, quando a dificuldade de se falar sobre a morte envolve desafios na comunicação entre paciente e profissional (Zimmermann \& Rodin, 2004). É necessário reduzir o sofrimento e maximizar a qualidade de vida, já que é impossível prolongar a vida ou obter a cura em todos os casos. No entanto, para que isto aconteça, é preciso que o profissional tenha conhecimento sobre o que constitui uma interação interpessoal adequada com a pessoa que está morrendo, que esteja apto emocionalmente para lidar com o luto antecipado da família e com seus próprios sentimentos de dor após a morte de um paciente (Weeber, 2005).

Assim, caberia aos profissionais dos sistemas de saúde prestar apoio técnico e instrumental, bem como conforto, apoio emocional e suporte para as incertezas, com habilidades específicas que estão subavaliadas no mundo acadêmico, como: bondade, paciência, empatia e reciprocidade. Habilidades contrárias àquelas mais desenvolvidas no modelo biomédico que privilegia uma formação profissional voltada para a eficiência e eficácia, ao invés de uma formação hábil de se envolver emocionalmente (Carpentier, 2013).

Abordando outra população, Solheim e Garratt (2013) analisaram o resultado da intervenção da aplicação de procedimentos técnicos em crianças internadas em hospitais. Uma maior satisfação dos pais em relação ao tratamento e às instalações hospitalares, como também as experiências anteriores com sua própria saúde e internações, interfeririam negativa ou positivamente em sua avaliação e participação no tratamento dos filhos. Concluem que o apoio aos pais e dos pais à criança durante o percurso do tratamento interfere positivamente para os resultados obtidos.

Embora haja uma ampliação de estudos que levaram em consideração que a racionalidade não é capaz de cobrir toda a esfera de forças na vida das pessoas, ainda acontecem majoritariamente estudos centrados na compreensão dos processos sociais pelo ângulo racional e cognitivo, mesmo enriquecidos com abordagens não quantitativas e interpretativas (Carpentier \& Grenier, 2012). Dessa maneira, em alguns estudos pôde-se observar uma resposta distanciada de intervenção para aquilo que foge ao foco da doença em questão, pois, mesmo considerando a presença de demandas subjetivas durante a aplicação de um determinado tratamento, as intervenções observadas foram sempre tecnicamente orientadas (Schouffoer et 
al., 2011; Turner et al., 2003). Este modelo de cuidado divide a atenção em especialidades distintas, cada uma cuidando do seu tipo de problema de saúde, de forma que a intervenção para as demandas subjetivas fica limitada à atenção psicológica ou psiquiátrica, não concebendo que possa haver algum tipo de participação de todos os profissionais envolvidos no acolhimento das necessidades do paciente.

\section{Práticas Integrativas e Complementares}

Os estudos que abordaram a utilização das Práticas Integrativas e Complementares (PIC) foram selecionados por trazerem uma perspectiva de cuidado em saúde que abrangia aspectos de subjetividade e, em sua ontologia, de sociabilidade.

O aumento do interesse pela utilização das PIC como alternativa de cuidado surgiu conjuntamente com os movimentos sociais relacionados com a New Age - movimento cultural nascido nas décadas de 1960 e 1970, que buscava uma maior integração entre as pessoas e a natureza, valorizando a busca pela espiritualidade e contestando as religiões tradicionais e a medicina científica, o que influenciou na adoção das chamadas "medicinas alternativas" (Barros, 2000) - e a perda de prestígio do modelo biomédico (Barros, 2006; Giddens, 2005; Luz, 2005), em diferentes populações mundiais (OMS, 2014).

Notou-se que o uso das PIC é uma expressão contemporânea do entrelaçamento do cuidado com a subjetividade, que surgiu da necessidade de encontrar outras formas de compreender o adoecimento e satisfazer a necessidade de cuidado. Como afirma Tesser (2007), independente da avaliação de qual terapêutica possuir a verdade diagnóstica e assumindo a possibilidade de haver mais de uma versão de diagnóstico para um mesmo adoecimento, é possível que mais de uma racionalidade terapêutica, mais de uma tradição especializada em saúde-doença-cuidado possam construir interpretações, oferecer coerência e veracidade, permitindo intervenção nos adoecimentos de diferentes formas com eficácia.

A insatisfação com o modelo biomédico mostrouse pelo descontentamento com o tratamento de doenças crônicas e com os medicamentos alopáticos; a busca por estilos de terapia mais holísticos e menos invasivos; a maior variedade de cuidados e a maior eficácia com muitos problemas de saúde; o valor mais barato de seus tratamentos; os resultados clínicos mais eficazes com sua adoção em conjunto com a prática convencional para conter problemas e erros da biomedicina; a resposta insatisfatória dada pelos profissionais (Freidin \& Timmermans, 2008; Gyasi et al., 2011; Isola, 2013; Saizar \& Korman, 2012 ; Shuval et al., 2012).

A presença de uma maior sociabilidade nos cuidados foi abordada pela oferta de um novo e melhor relacionamento entre o profissional e o usuário; o favorecimento de um papel central e responsabilidade do indivíduo em seus cuidados; a facilidade de acesso aos cuidadores que prestam serviço à comunidade; a maior integração à cultura local; o apoio à preferência individual em ter responsabilidade por sua saúde; a familiaridade com tratamentos alternativos no próprio contexto social; a visão de que a biologia e a cultura interagem como parceiras iguais na definição de quem e o que somos (Freidin \& Timmermans, 2008; Gyasi et al., 2011; Isola, 2013; Saizar \& Korman, 2012 ; Shuval et al., 2012).

A atenção a aspectos subjetivos nos cuidados foi explicitada pela ajuda ao paciente para lidar com o stress surgido após um diagnóstico de câncer; a oferta de um tratamento individualizado; o maior conforto psicológico dos pacientes com o seu uso; o desenvolvimento de consciência e compreensão individual do processo do adoecimento e de comportamentos preventivos; a transformação pessoal e autorrealização; a compreensão própria da segurança e eficácia dessas alternativas; a doença vista como um desequilíbrio entre forças opostas de energia e de uma falha do poder restaurador do corpo (Daykin, McClean, \& Bunt, 2007; Freidin \& Timmermans, 2008; Gyasi et al., 2011; Shuval et al., 2012).

\section{Conclusão}

Os resultados apresentados por esta revisão sistemática da literatura não são inovadores, na medida em que tratam de aspectos já discutidos, contudo explicitam diferentes preocupações com a insatisfação com a forma de intervenção em saúde, o que pode postular que há uma crise do cuidado.

As discussões sobre As limitações do cuidado biomédico demonstraram que, no desenvolvimento da clínica biomédica, o significado do substantivo cuidado foi naturalizado como aplicação técnica e tecnológica de procedimentos na esfera da saúde, com um maior reconhecimento social de sua especialização. Como consequência, houve um afastamento do seu sentido etimológico, que se refere a um ato feito com zelo, de atenção diferenciada por estar carregada de preocupação afetiva e, principalmente, de ser compreendido na sua dimensão social, de solidariedade entre pessoas que se unem pela manutenção da vida. Na contemporaneidade, 
mudanças estão sendo concebidas visando à redefinição e qualificação das ações de cuidado, especialmente por compreender as maneiras como são expressas as subjetividades e, em seu interior, as sociabilidades implícitas em qualquer interação social.

Já os elementos trazidos em $A$ sociabilidade nas ações de cuidado explanam que o cuidado não pode mais ser visto apenas na sua forma aplicada. Ele deve ser compreendido na dimensão interacional entre pessoas, e é desse lugar que emergem os aspectos positivos ou negativos da sua qualificação. As redes sociais foram constatadas como fundamentais para $\mathrm{o}$ resultado das ações do cuidado, pois possibilitaram a compreensão de que o cuidado e a sociabilidade estão intrinsecamente relacionados e que as ações em indivíduos isolados pautadas apenas na objetividade técnica não oferecem os melhores resultados.

Em diálogo, as considerações encontradas em $O$ entrelaçamento entre cuidado e subjetividade demonstram que a busca pelo cuidado não está somente na aplicação técnica/tecnológica profissionalizada: também está na interação com alguém significativo, que dê suporte, acolhimento e empatia, num momento de perda do controle de si e de angústia, que acompanham o adoecimento. $\mathrm{O}$ que demanda profissionais qualificados para tal, uma vez que, conforme relatam os textos, há a percepção pelos pacientes do esvaziamento das ações técnicas de cuidado. Estudos descreveram um sentimento de insegurança diante da ajuda oferecida, que não foi capaz de oferecer acolhimento ao sofrimento diante do adoecimento, ou dar um suporte coletivo na busca cotidiana pelo bem-estar. O que leva à conclusão de que a subjetividade implícita no adoecer não tem sido contemplada adequadamente pelo campo da saúde.

Por sua vez, os textos sobre as Práticas Integrativas e Complementares descreviam processos na busca de valorização de aspectos subjetivos, com suas sociabilidades envolvidas, nas ações de cuidado desenvolvidas.

Infere-se, portanto, que apenas o uso da técnica e da tecnologia não dão substância ao cuidado, pois a solidariedade, o acolhimento e a empatia devem ser compreendidos como recursos fundamentais na construção de sociabilidades cuidadoras e subjetividades emancipadoras na atenção à saúde.

\section{Referências}

Andersen, R. S. et al. (2011). Does the organizational structure of health care systems influence care-seeking decisions? A qualitative analysis of Danish cancer patients' reflections on care-seeking. Scandinavian Journal of Primary Health Care, 29(3), 144-149.

Bahar, Z. et al. (2005). The Effects of Islam and Traditional Practices on Women's Health and Reproduction. Nursing Ethics, 12(6), 557-570.

Barros, N. F. (2000). Medicina complementar uma reflexão sobre o outro lado da prática. São Paulo: Annablume/ FAPESP.

Barros, N. F. (2006). A construção da medicina integrativa. São Paulo: Hucitec.

Basu, A. M. (2006). The Emotions and Reproductive Health. Population and Development Review, 32(1), 107-121.

Boltanski, L. (2004). As classes sociais e o corpo. Rio de Janeiro: Graal.

Brown, P. \& Flores, R. (2011). Making normative structures visible: The British National Health Service and the hospice movement as signifiers of compassion and hope. Acta Sociologica, 54(1), 61-75.

Burkhart, L. \& Hogan, N. (2008). An Experiential Theory of Spiritual Care in Nursing Practice. Qualitative Health Research, 18(7), 928-938.

Camargo, K. R. (2005). A biomedicina. PHYSIS: Rev. Saúde Coletiva, 15(Supl.), 177-201.

Campos, G. W. S. \& Bedrikow, R. (2014). História da Clínica e Atenção Básica - o desafio da ampliação. São Paulo: Huitec.

Canguilhem, G. (2002). O normal e o patológico. Rio de Janeiro: Forense Universitária.

Carpentier, N. \& Grenier, A. (2012). Successful Linkage Between Formal and Informal Care Systems: The Mobilization of Outside Help by Caregivers of Persons With Alzheimer's Disease. Qualitative Health Research, 22(10), 1330-1344.

Carpentier, N. (2013). Entry Into a Care Trajectory: Individualization Process, Networks, and the Emerging Project. SAGE Open, 3(2), 1-9.

Castel, R. (1998). As metamorfoses da questão social. Petrópolis, RJ: Vozes.

Daykin, N., McClean, S., \& Bunt, L. (2007). Creativity, identity and healing: participants' accounts of music therapy in cancer care. Health, 11(3), 349-370.

Denier, Y. (2008). Mind the gap! Three approaches to scarcity in health care. Medicine, Health Care and Philosophy, 11(1), 73-87.

Dovidio, J. F. et al. (2008). Disparities and distrust: The implications of psychological processes for understanding racial disparities in health and health care. Social Science and Medicine, 67(3), 478-486.

Edwards, C. Staniszweska, S., \& Crichton, N. (2004). Investigation of the ways in which patients' reports of their satisfaction with healthcare are constructed. Sociology of Health and Illness, 26(2), 159-183.

Farrant, B. \& Watson, P. D. (2004). Health care delivery: Perspectives of young people with chronic illness and their parents. Journal of Paediatrics \& Child Health, 40(4), 175179.

Ferlander, S. (2007). The Importance of Different Forms of Social Capital for Health. Acta Sociologica, 50(2), 115-128.

Fine, M. (2005). Individualization, risk and the body: Sociology and care. Journal of Sociology, 41(3). 247-266.

Foucault, M. (1977). O nascimento da clínica. Rio de Janeiro: Forense Universitária.

Freidin, B. \& Timmermans, S. (2008). Complementary and Alternative Medicine for Children's Asthma: Satisfaction, 
Care Provider Responsiveness, and Networks of Care. Qualitative Health Research, 18(1), 43-55.

Frúgoli, H. (2007). Sociabilidade urbana. Rio de Janeiro: Jorge Zahar.

Giddens, A. (2005). Sociologia (4a ed.). Porto Alegre: Artmed.

González Rey, F. (2007). As categorias de sentido, sentido pessoal e sentido subjetivo: sua evolução e diferenciação na teoria histórico-cultural. Psicologia da Educação, 24, 155179.

Greenhalgh, T. Robb, N., \& Scambler, G. (2006). Communicative and strategic action in interpreted consultations in primary health care: A Habermasian perspective. Social Science and Medicine, 63(5), 1170-1187.

Gyasi, R. M. et al. (2011). Public Perceptions of the Role of Traditional Medicine in the Health Care Delivery System in Ghana. Global Journal of Health Science, 3(2), 40-49.

Hudson, P. L. et al. (2006). Responding to desire to die statements from patients with advanced disease: recommendations for health professionals. Palliative Medicine, 20(7), 703-710.

Illich, I. (1975). A expropriação da saúde: nêmeses da medicina. Rio de Janeiro: Nova Fronteira.

Isola, O. I. (2013). The "relevance" of the african traditional medicine (alternative medicine) to health care delivery system in Nigeria. The Journal of Developing Areas, Nashville, 47(1), 319-338.

Levy, A. R. (2005). Categorizing Outcomes of Health Care Delivery. Clinical and Investigative Medicine, 28(6), 347-50.

Lopez, S. H. (2006). Emotional Labor and Organized Emotional Care: Conceptualizing Nursing Home Care Work. Work and Occupations, 33(2), 133-160.

Luz, M. T. (2005). Cultura contemporânea e medicinas alternativas: novos paradigmas em saúde no fim do século XX. Physis: Rev. Saúde Coletiva, 15(Supl.), 145-176.

Noone, J. H. \& Stephens, C. (2008). Men, masculine identities, and health care utilisation. Sociology of Health and Illness, 30(5), 711-725.

Organização Mundial da Saúde. (2014). Tradicional Medicine Strategy. Recuperado de http://apps.who.int/iris/ bitstream/10665/92455/1/9789241506090_eng.pdf?ua=1.

Parsons T. (1951). The social system. Glencoe, IL: Free Press.

Pescosolido, B. A. (2006). Of Pride and Prejudice: The Role of Sociology and Social Networks in Integrating the Health Sciences. Journal of Health and Social Behavior, 47(3), 189208.

Prior, L. (2003). Belief, knowledge and expertise: the emergence of the lay expert in medical sociology. Sociol Health Illn, 25, 41-57.

Saizar, M. \& Korman, G. (2012). Interactions Between Alternative Therapies and Mental Health Services in Public Hospitals of Argentina. SAGE Open, 2(3), 1-13.

Salvalaggio, G. et al. (2013). Patient-Provider Rapport in the Health Care of People Who Inject Drugs. SAGE Open, 3(4), $1-13$.

Santos, B. S. (2002). Um discurso sobre as ciências. Porto, PORT: Afrontamento.

Santos, G. C. S. \& Martínez, A. M. A. (2016). Subjetividade social da escola e os desafios da inclusão de alunos com desenvolvimento atípico. Revista Brasileira de Educação Especial, 22(2), 253-268.

Schouffoer, A. A. et al. (2011). Needs and preferences regarding health care delivery as perceived by patients with systemic sclerosis. Clinical Rheumatology, 30(6), 815-824.
Shuval, J. T. et al. (2012). Integrating CAM and Biomedicine in Primary Care Settings: Physicians' Perspectives on Boundaries and Boundary Work. Qualitative Health Research, 22(10), 1317-1329.

Smith, G. C. (2009). From Consultation-Liaison Psychiatry to Integrated Care for Multiple and Complex Needs. Australian and New Zealand Journal of Psychiatry, 43(1), 1-12.

Solheim, E. \& Garratt, A. M. (2013). Parent experiences of inpatient pediatric care in relation to health care delivery and sociodemographic characteristics: results of a Norwegian national survey. BMC Health Services Research, 13, 1-12.

Sorensen, R. \& Iedema, R. (2008). Redefining accountability in health care: Managing the plurality of medical interests. Health, 12(1), 87-106.

Spates, K. (2012). “The Missing Link”: The Exclusion of Black Women in Psychological Research and the Implications for Black Women's Mental Health. SAGE Open, 2(3), 1-8.

Tesser, C. D. (2007). A verdade na biomedicina, reações adversas e efeitos colaterais: uma reflexão introdutória. PHYSIS: Rev. Saúde Coletiva, 17(3), 465-484.

Turner, B. J. M. D. M. et al. (2003). Relationship of gender, depression, and health care delivery with antiretroviral adherence in HIV-infected drug users. Journal of General Internal Medicine, 18(4), 248-257.

Wajid, A. White, F., \& Karim, M. S. (2013). Community Health Workers and Health Care Delivery: Evaluation of a Women's Reproductive Health Care Project in a Developing Country. PLoS One, 8(9), 1-10.

Weeber, S. C. (2005). Death, Grief, and the Home Health Worker: A Systems Approach. Home Health Care Management \& Practice, 17(5), 358-364.

Zimmermann, C. \& Rodin, G. (2004). The denial of death thesis: sociological critique and implications for palliative care. Palliative Medicine, 18(2), 121-28.

Submissão em: 17/03/2017

Revisão em: 10/11/2017

Aceite em: 19/12/2017

\section{Agradecimento}

À Agência de fomento, Coordenação de Aperfeiçoamento de Pessoal de Nível Superior CAPES. Processo no. 01P-03490/2014.

Octávio Augusto Contatore é psicólogo, mestre e doutorando em Saúde Coletiva pela Universidade Estadual de Campinas, SP. https://orcid.org/0000-0003-3486-7924

Endereço: Rua Tessália Vieira de Camargo, 126, Cidade Universitária Zeferino Vaz. CEP 13083-887, Campinas/SP.

E-mail: oacontatore@yahoo.com.br

Ana Paula Serrata Malfitano é professora Associada do Departamento de Terapia Ocupacional e do Programa de pós-graduação em Terapia Ocupacional da Universidade 
Federal de São Carlos, SP. Doutora em Saúde Pública pela Universidade de São Paulo, SP. https://orcid.org/0000-0002-0502-3194

E-mail: anamalfitano@ufscar.br

Nelson Filice de Barros é professor Livre-docente do Departamento de Saúde Coletiva e do Programa de pós- graduação em Saúde Coletiva da Faculdade de Medicina da Universidade Estadual de Campinas, SP. Coordenador do Laboratório de Práticas Alternativas, Complementares e Integrativas em Saúde. https://orcid.org/0000-0002-5404-1516 E-mail: filice@,fcm.unicamp.br 